\title{
CARACTERIZACIÓN DEL TAMAÑO DE SEMILLA NODRIZA DE AGUACATE ESTHER Y EL DESARROLLO DE SUS PLÁNTULAS
}

\section{CHARACTERIZATION OF AVOCADO NURSE SEED SIZE AND SEEDLING DEVELOPMENT IN CV. ESTHER}

\author{
Luisa Gálvez-Cendegui, Patricia Peñaloza, \\ Eduardo Oyanedel y Mónica Castro*
}

Escuela de Agronomía, Pontificia Universidad Católica de Valparaíso. Casilla 4-D, Quillota, Chile. Tel: (56)-32-2274530 y Fax: (56)-32-2274570

*Autor para correspondencia (monica.castro@pucv.cl)

\section{RESUMEN}

La propagación de aguacate (Persea americana Mill.) mediante las técnicas de etiolación y acodo, requiere semillas nodriza que generen plántulas homogéneas y con determinado diámetro para injertación en el menor tiempo posible. El tamaño de las semillas suele usarse como criterio de selección. Este estudio caracterizó físicamente tres categorías de peso fresco de semilla de aguacate cv. Esther; se determinó la correlación entre el peso fresco del fruto y el peso fresco de la semilla; y se evaluó el efecto del peso fresco de la semilla sobre el tiempo de crecimiento de la plántula. La investigación se llevó a cabo en las temporadas 2009 y 2013. En la temporada 2009 se clasificó a 120 semillas elegidas al azar en tres categorías de peso fresco: pequeño (28 a $43 \mathrm{~g}$ ), mediano ( 44 a $59 \mathrm{~g}$ ) y grande ( 60 a $75 \mathrm{~g}$ ), y se compararon sus características físicas (diámetro, perímetro y peso fresco del eje embrionario). En ambas temporadas se pesaron 90 frutos y sus correspondientes semillas, se correlacionaron el peso fresco de los frutos y semillas. En ambas temporadas se separaron por sus pesos 45 semillas en las mismas categorías de peso fresco de la caracterización física, y se sembraron en condiciones de invernadero con calefacción (temperatura media $28 \pm 5^{\circ} \mathrm{C}$ ). Se midió el tiempo que tardó en emerger la plúmula y el requerido para que los tallos alcanzaran $4.5 \mathrm{~mm}$ de diámetro y $20 \mathrm{~cm}$ de longitud. Las semillas grandes presentaron mayor diámetro, perímetro y peso fresco del eje embrionario que las pequeñas. Se determinó una correlación positiva significativa entre el tamaño de frutos y semillas en las dos temporadas de evaluación. No hubo efecto del peso fresco de la semilla sobre el tiempo de crecimiento de las plántulas en ambas temporadas.

Palabras clave: Persea americana, biomasa de eje embrionario, calidad de semilla, peso fresco de semilla, vigor de semilla.

\section{SUMMARY}

Avocado (Persea americana Mill.) propagation by etiolation and layering requires nurse seeds that generate homogeneous plantlets, capable of attaining the minimum diameter required for grafting in the shortest time. Seed size is often used as selection criterion. This study characterized three groups of seeds sorted by fresh weight, in the cv. Esther; the correlation between fruit and seed fresh weights was determined; and the effect of seed fresh weight on seedling growth was evaluated. The research was conducted in the 2009 and 2013 seasons. In 2009, 120 randomly selected seeds were sorted into three classes: small ( 28 to $43 \mathrm{~g}$ ), medium ( 44 to $59 \mathrm{~g}$ ) and large (60 to $75 \mathrm{~g}$ ), and their physical traits (diameter, perimeter and embryo axis fresh weight) were compared. In both seasons, 90 fruits and their seeds were weighed, and both fresh weights were correlated; also 45 seeds were separated by their weights in the above mentioned categories and sown in a heated greenhouse (mean temperature $28 \pm 5^{\circ} \mathrm{C}$ ). Periods required for plumule emergence and for the stems to reach $4.5 \mathrm{~mm}$ in diameter and $20 \mathrm{~cm}$ stem length were registered. Large seeds showed higher diameter, perimeter and embryo axis fresh weight than small seeds. In both seasons a positive correlation between fruit and seed sizes was determined, although no effect of seed fresh weight over seedling growth rate was found.

Index words: Persea americana, biomass embryo axis, fresh weight of seed, seed quality, seed vigor.

\section{INTRODUCCIÓN}

En la propagación clonal de aguacate (Persea americana Mill.) mediante la técnica de etiolación y acodo, la semilla nodriza es la encargada de nutrir a la plántula y posteriormente al portainjerto clonal, mientras se lleva a cabo el desarrollo de raíces adventicias. Este proceso de nutrición finaliza en el primer año de vida del portainjerto en el huerto (Castro y Fassio, 2013; Ernst et al., 2013). Habitualmente, las semillas se seleccionan para obtener plántulas homogéneas, que posean un tallo que supere los $4.5 \mathrm{~mm}$ de diámetro y los $20 \mathrm{~cm}$ de longitud al momento de ser injertadas. En general, se prefieren las semillas de tamaño grande porque contienen gran cantidad de carbohidratos, aceites y proteínas de reserva (Ceballos y Montoya, 2013; García-Fajardo et al., 1999; Olaeta et al., 2007). Estos compuestos están disponibles para sustentar el crecimiento de la plántula en los estados tempranos, antes de convertirse en una planta independiente, autotrófica, capaz de usar la energía de la luz (Bewley et al., 2013) y durante todo el proceso de desarrollo de la raíz (Castro y Fassio, 2013).

Algunos propagadores seleccionan variedades de semilla grande para obtener plantas con diámetro de injertación en menor tiempo. Waldin, Lula, Zutano, Reed y Ettinger son algunos cultivares específicos de aguacate que se han utilizado como semilla nodriza. Las semillas de Lula se caracterizan por tener tamaño pequeño en comparación con Waldin, y generan un bajo vigor de la plántula. Sin embargo, al comparar Lula con Zutano en cuanto a su comportamiento como semilla nodriza, Lula le da más vigor al crecimiento del brote lo que permite injertar la variedad comercial en menos tiempo (Whiley y Anderson, 2002). Otras variedades de aguacate de gran tamaño de semilla que se podrían 
usar para estos fines son Gwen (64 g), Panchoy (61 g), Whitsel (60 g), Benik (52 g), Esther (47 g) y Carlsbad (33 g) (Navarro, 2005; Com. pers. ').

Al evaluar en México semillas de aguacate criollo y del cultivar Hass, Flores et al. (1990) determinaron que los pesos fresco y seco promedio de la semilla fueron mayores en Hass. Además, determinaron que las semillas de criollos fueron las más tempranas en emitir la plúmula. De este estudio se concluye que las semillas de cultivares de mayor tamaño tienden a demorarse más en emitir la plúmula.

De igual forma, Olivares (2004; Com. pers. ${ }^{2}$ ) estudió algunas características de los cultivares Rincón, Nabal, Edranol y Hass, enlistadas en orden de mayor a menor peso fresco de semilla. Con excepción de Nabal, al comparar estas variedades se observó una correlación positiva entre el tamaño de la semilla y la tasa de crecimiento de la plántula. A pesar de que Nabal no fue la de mayor peso, presentó la mayor tasa de crecimiento en longitud y diámetro de plántula, que llegaron a valores de $25.7 \mathrm{~cm}$ y $4.4 \mathrm{~mm}$ respectivamente, en menos tiempo que los otros cultivares. Esto indica que las variedades de aguacate con semillas de mayor peso tendrían mayor tasa de crecimiento de plántula que las variedades de semillas más pequeñas, pero esta relación no siempre se cumpliría dada la influencia del genotipo. En otras especies anuales como maíz (Zea mays L.) también se ha observado que las semillas de mayor tamaño originaron plántulas más grandes, lo que se atribuye al potencial genético de la variedad y al contenido de reservas de la semilla (Pérez et al., 2006; Sulewska et al., 2014).

Medina (2007; Com. pers. ${ }^{3}$ ) también evaluó el peso de la semilla de algunas variedades con potencial de uso como semilla nodriza, tales como Ettinger, Rincón, Zutano, Carlsbad, Panchoy y Nabal. En este estudio, Panchoy (94.4 g) fue de mayor peso que Carlsbad (43.3 g) y ambas a su vez de mayor peso que Nabal. Panchoy demoró más en emitir la radícula que Carlsbad, lo cual se explicaría por la mayor distancia que tiene que recorrer la radícula al interior de la semilla. Sin embargo, Panchoy presentó mayor tasa de crecimiento que Carlsbad a partir de la emergencia de su plúmula, y demoró menos tiempo en alcanzar el diáme-

\footnotetext{
'Navarro M. (2005) Formación y evaluación del banco de germoplasma de variedades y portainjertos de palto (Persea americana Mill.). Estación Experimental La Palma. 136 p. Taller de Licenciatura Ing. Agrónomo. Pontificia Universidad Católica de Valparaíso. Quillota, Chile. 2Olivares D. (2004) Implementación de la técnica de propagación clonal del palto (Persea americana Mill.): Uso de semillas nodrizas. 85 p. Taller de Licenciatura Ing. Agrónomo. Pontificia Universidad Católica de Valparaíso. Quillota, Chile.

${ }^{3}$ Medina G. (2007) Efecto de diferentes factores de la semilla nodriza sobre la propagación clonal de paltos (Persea americana Mill.) mediante la técnica de etiolación y acodo en invernadero climatizado. 45 p. Taller de Licenciatura Ing. Agrónomo. Pontificia Universidad Católica de Valparaíso. Quillota, Chile.
}

tro requerido para injertar. En algunas especies del género Quercus, el tamaño de semilla se relacionó con la cantidad de reservas y el peso de la plántula, e influyó también el progenitor. Entonces, tanto la genética del progenitor como el tamaño de la semilla influirían en la biomasa de la plántula (González et al., 2008).

Por otra parte, al comparar el efecto del peso de semillas Ettinger y Zutano sobre la tasa de crecimiento de las plántulas, se observó que las semillas más grandes obtuvieron antes el diámetro de injertación, aun cuando ocurrieron en diferente momento del desarrollo de las plántulas según cada variedad (Bozzolo, 2006; Com. pers. ${ }^{4}$; Medina, 2007; Com. pers. $\left.{ }^{3}\right)$. Por lo tanto, el tamaño de la semilla influiría en gran medida en la tasa de crecimiento de la planta nodriza. En otras especies herbáceas como maíz, frijol (Phaseolus vulgaris L.) y tabaco (Nicotiana tabacum L.), se observó que las semillas grandes y medianas tuvieron un crecimiento superior que las pequeñas (Barrios et al., 2014; García-Almañy y Lobo, 2007; Laynez et al., 2007; TadeoRobledo et al., 2010).

En Chile, la semilla nodriza que ha mostrado los mejores resultados en clonación de aguacate pertenece a la variedad Esther; sin embargo, en la primera etapa de clonación del aguacate se ha observado crecimiento desuniforme de las plántulas. En un vivero es de importancia la utilización óptima del espacio, con el fin de obtener plantas homogéneas, de alta calidad y en el menor tiempo posible (Spann y Ferguson, 2014). No existen estudios que describan físicamente a las semillas de aguacate Esther, ni el comportamiento de esta variedad como semilla nodriza.

De acuerdo con la información recabada en aguacate y en otras especies, se establece la siguiente hipótesis: las características físicas de la semilla como su peso fresco, diámetro, perímetro y peso fresco del eje embrionario afectarían el crecimiento de las plántulas. El peso fresco de la semilla estaría relacionado con el peso fresco del fruto. En otras especies se ha reportado que existe relación positiva entre el tamaño de la semilla, el tamaño del eje embrionario y el crecimiento de las plántulas (Barrios et al., 2014; García-Almañy y Lobo, 2007; González et al., 2008; Laynez et al., 2007; Tadeo-Robledo et al., 2010).

Los objetivos de este estudio fueron caracterizar físicamente tres categorías de semilla nodriza Esther en cuanto a diámetro, perímetro y peso fresco del eje embrionario; determinar la correlación entre el peso fresco del fruto y el peso fresco de la semilla; y evaluar el efecto del peso fresco de la

${ }^{4}$ Bozzolo R. (2006) Implementación de la técnica de etiolación y acodo en la propagación clonal de palto (Persea americana Mill.): Etapa de enraizamiento. 45 p. Taller de Licenciatura Ing. Agrónomo. Pontificia Universidad Católica de Valparaíso. Quillota, Chile. 
semilla sobre el desarrollo de la plántula.

\section{MATERIALES Y MÉTODOS}

\section{Material vegetal, condiciones experimentales y variables evaluadas}

Este ensayo se llevó a cabo en las temporadas 2009 y 2013 en el Laboratorio de Propagación de la Escuela de Agronomía, Pontificia Universidad Católica de Valparaíso, ubicada en la provincia de Quillota $\left(32^{\circ} 50^{\prime} \mathrm{S} ; 71^{\circ} 13^{\prime} \mathrm{O}\right.$, a una altitud de $120 \mathrm{~m}$ ), región de Valparaíso, Chile. Las semillas de la variedad Esther utilizadas en ambas temporadas se obtuvieron de un huerto ubicado en la localidad de Pedegua, Provincia de Petorca, Región de Valparaíso, Chile ( $32^{\circ} 18^{\prime} \mathrm{S} ; 71^{\circ} 04^{\prime} \mathrm{O}$; a una altitud de $330 \mathrm{~m}$ ). Este huerto tiene una superficie de 3 ha y además posee árboles de la variedad Hass (10 \%) como donadores de polen. Los frutos se cosecharon en forma individual desde distintos sectores de los árboles, previa determinación del porcentaje de materia seca (mayor a $20 \%$ ). Una vez cosechados los frutos se mantuvieron a temperatura ambiente hasta el ablandamiento de la pulpa, tras lo cual se extrajo la semilla, se eliminaron los restos de pulpa mediante lavados sucesivos con agua, para finalmente secarlas a la sombra durante $24 \mathrm{~h}$.

Del lote de semillas de la temporada 2009 se pesaron 120 unidades en una balanza de precisión (Precisa 620 C, Suiza), y conforme a su peso fresco se separaron en tres categorías: pequeño (28 a 43 g), mediano (44 a 59 g) y grande (60 a $75 \mathrm{~g}$ ). Se les realizó un corte longitudinal para evaluar las características físicas de las semillas: largo, ancho y perímetro. Para ello se digitalizaron imágenes con un escáner (Hewlett Packard®, Scanjet 7450c, Flatbed), posteriormente se extrajeron los datos desde las imágenes con el programa SIGMA SCAN PRO versión 5.0, mediante las herramientas disponibles (Systat Software Inc., SigmaScan ${ }^{\circledR}$ Pro image analysis software). Además, se registró el peso fresco del eje embrionario.

Por otra parte, se midió el peso fresco de 90 frutos y sus correspondientes semillas en las temporadas 2009 y 2013. Posteriormente estas semillas se separaron en las mismas tres categorías de peso fresco antes mencionadas. Se escarificaron mecánicamente en la parte basal mediante un raspado superficial. Luego se trataron con una mezcla de $1.8 \mathrm{~g} \mathrm{~L}^{-1}$ de Benomyl (i. a.) y $1.8 \mathrm{~g} \mathrm{~L}^{-1} \mathrm{de}$ Captan (i. a.) durante $10 \mathrm{~min}$. Se sembraron en cajones con sustrato de turba y perlita 1:1 (v:v) para realizar el proceso de pre-germinación en sustrato húmedo. Una vez que las semillas presentaron radícula igual o superior a $1 \mathrm{~cm}$, se trasplantaron a contenedores de polietileno de $1 \mathrm{~L}$ de capacidad rellenado con sustrato Sunshine Mix No3 (SUN
GRO®, Canadá), previamente regados. La siembra se hizo en condiciones de invernadero con calefacción, con una temperatura media de $28 \pm 5^{\circ} \mathrm{C}$.

Finalmente, se registró el tiempo (días) que las semillas demoraron en emitir la plúmula, los días en alcanzar el diámetro de injertación correspondiente a $4.5 \mathrm{~mm}$ y los días que demoró el tallo en crecer hasta $20 \mathrm{~cm}$ de longitud. Para ello fue necesario medir el diámetro $(\mathrm{mm})$ a una altura de $3 \mathrm{~cm}$ desde el cuello de la plántula, y la longitud de tallo $(\mathrm{cm})$ desde el cuello hasta el ápice caulinar de la plántula cada $7 \mathrm{~d}$.

\section{Diseño y análisis estadístico}

Se utilizó un diseño completamente al azar univariado, donde los tratamientos fueron los tres tamaños de la semilla nodriza. Las variables de respuesta fueron las características físicas de frutos y semillas y el tiempo (días) para alcanzar el diámetro de injertación. Los datos fueron analizados con un modelo estadístico completamente aleatorio. Cuando el análisis detectó diferencias entre tratamientos se aplicó la prueba de Tukey $(P \leq 0.05)$ para la separación de medias. La correlación de Pearson se usó para establecer la asociación entre las características físicas de la semilla y los pesos frescos de frutos y semillas. La información se analizó con el sistema estadístico MINITAB versión 16.0 (Minitab Inc.).

\section{RESULTADOS Y DISCUSIÓN}

\section{Caracterización física de las semillas}

Tanto diámetro, como perímetro y peso fresco del eje embrionario fueron diferentes $(P \leq 0.05)$, según las categorías de peso analizadas (Cuadro 1). Las semillas de categoría grande tuvieron un mayor peso fresco del eje embrionario que las dos categorías más pequeñas $(P \leq 0.05)$. Debido a la escasez de información reciente que asocie las características físicas de las semillas de aguacate con el desarrollo de la plántula, como referencia se utilizaron trabajos realizados en otras especies. En frijol, las semillas grandes presentaron mayor biomasa de embrión que las semillas medianas y pequeñas (Barrios et al., 2014), al igual que lo observado en este ensayo.

La semilla de aguacate conforma una potente demanda que atrae fotoasimilados (almidón, proteínas y lípidos), a través de los cuales también crece el pericarpio del fruto (Blumenfeld y Gazit, 1974; Sánchez-Romero et al., 2002). Ortega-David et al. (2010) y Sánchez et al. (2011) señalaron que en especies arbóreas de un bosque tropical siempre verde y en especies herbáceas como lupino (Lupinus mutabilis Sweet.), el tamaño de 
Cuadro 1. Caracterización física de tres categorías de semilla de aguacate cv. Esther. Valparaíso, Chile, 2009. $(n=120)$.

\begin{tabular}{lcccc}
\hline PFS $(\mathrm{g})$ & DLS $(\mathrm{cm})$ & DES $(\mathrm{cm})$ & PMS $(\mathrm{cm})$ & PFE $(\mathrm{g})$ \\
\hline Pequeño (28 a 43) & $3.90 \mathrm{c} \mathrm{c}^{*}$ & $3.91 \mathrm{c}$ & $13.14 \mathrm{c}$ & $0.15 \mathrm{~b}$ \\
Mediano (44 a 59) & $4.38 \mathrm{~b}$ & $4.35 \mathrm{~b}$ & $14.70 \mathrm{~b}$ & $0.17 \mathrm{ab}$ \\
Grande (60 a 75) & $4.77 \mathrm{a}$ & $4.77 \mathrm{a}$ & $16.10 \mathrm{a}$ & $0.21 \mathrm{a}$ \\
\hline
\end{tabular}

*Medias con letras iguales en cada columna no son estadísticamente diferentes (Tukey, 0.05). PFS = peso fresco de la semilla; DLS = diámetro longitudinal de la semilla; DES = diámetro ecuatorial de la semilla; PMS = perímetro de la semilla; PFE = peso fresco del eje embrionario.

la semilla estaría relacionado con la capacidad de fotoasimilados que puede almacenar, por lo que semillas más grandes tendrían mayor contenido nutricional.

\section{Relación entre peso del fruto y peso de la semilla}

La correlación entre el peso fresco de la semilla y el fruto de Esther fue positiva y baja $(P \leq 0.05)$ en la temporada $2009(r=0.75)$ y en la temporada $2013(r=0.60)$ (Figura $1)$, resultados que son similares a lo antes observado en Nabal $(r=0.60)$, Rincón ( $r=0.64)$ y Zutano $(r=0.61)$ (Medina, 2007; Com. pers. ${ }^{3}$ ). La diferencia en la correlación entre el peso fresco del fruto y la semilla entre cada temporada analizada se debe a que el tamaño del fruto está controlado por diversos factores genéticos y ambientales (Schaffer et al., 2013).

De acuerdo con estudios realizados por Degani et al. (1990), la variedad del donador de polen influye en los tamaños del fruto, del pericarpio y de la semilla de aguacate. En el presente ensayo se utilizaron semillas provenientes de un huerto combinado con Hass, lo que puede favorecer semillas de tamaños heterogéneos. Por su parte, Cowan et al. (2001) señalaron que el tamaño del fruto es consecuencia de un complejo proceso metabólico entre la fertilización del óvulo y el desarrollo del fruto, donde el tamaño de éste depende del número de células que logra, más que del tamaño de las mismas, y por lo tanto también depende de factores que afecten el ciclo de división celular, como las condiciones ambientales, entre otras. Las fitohormonas juegan un papel importante en este proceso metabóli$\mathrm{co}$, ya que mediante ellas se atraen los fotoasimilados que nutren tanto a la semilla como al fruto (Blumenfeld y Gazit, 1974; Zhang et al., 2007). Otro factor determinante en el tamaño es la ubicación del fruto en el brote (Finazzo et al., 1992).

\section{Desarrollo de las plántulas provenientes de tres categorías de tamaños de semilla}

No hubo efecto del tamaño de la semilla sobre las variables evaluadas en las plántulas de aguacate en ambas temporadas (2009 y 2013) (Figuras 2 y 3), excepto en el tiempo promedio que tardaron las semillas en emitir la plúmula en el ensayo del 2013, en la que se observó que las semillas grandes con peso fresco superior a $60 \mathrm{~g}$ fueron más tardías que las semillas medianas $(P \leq 0.05)$. En estudios realizados con la variedad Zutano se observó un comportamiento similar, ya que las semillas grandes fueron las que obtuvieron mayor tasa de crecimiento a partir de la emisión de la plúmula, pero no hubo diferencia en el tiempo total entre siembra y diámetro de injertación (Medina, 2007; Com. pers.3).

En ambas temporadas no hubo efecto del peso fresco de la semilla sobre las variables de crecimiento evaluadas. Desde el punto de vista del vigor de la semilla (AOSA, 1983; ISTA, 1995), es factible que éste exprese diferencias cuando el desarrollo de las plántulas ocurre en condiciones ambientales adversas, situación contraria a la que se produjo durante la investigación realizada. Por otra parte, tanto los factores internos de la semilla como las características genéticas de cada embrión, restarían el efecto del tamaño de la semilla sobre el desarrollo de las plántulas, debido a la alta heterocigosis que caracteriza a esta especie (Calabrese, 1992; Castro et al., 2003; Lahav y Lavi, 2015). En otras especies, como las pertenecientes al género Quercus, González et al. (2008) determinaron la importancia de la selección de semillas para la obtención de plantas en vivero, con base en el tamaño de las mismas y la variabilidad introducida por el progenitor.

El resultado obtenido en todas las variables de crecimiento (Figuras 2 y 3) fue opuesto a lo observado en otras especies de semillas diferentes en tamaño y composición nutricional como tabaco, maíz y frijol. García-Almañy y Lobo (2007) observaron que las semillas medianas y grandes de tabaco lograron mayor uniformidad de germinación y crecimiento de las plántulas en comparación con las pequeñas. También Tadeo-Robledo et al. (2010) observaron que las semillas grandes de maíz originaron plántulas con mayor peso seco que las semillas pequeñas. Barrios et al. (2014) señalaron que semillas más grandes de frijol mostraron mayor crecimiento inicial de las plántulas.

Al comparar ambas temporadas de medición se detectó un crecimiento variable, ya que las plántulas de la temporada 2009 (Figuras 1 y 2) tardaron más en crecer $(P \leq 0.05)$. Esta diferencia en el crecimiento de las plántulas estaría 

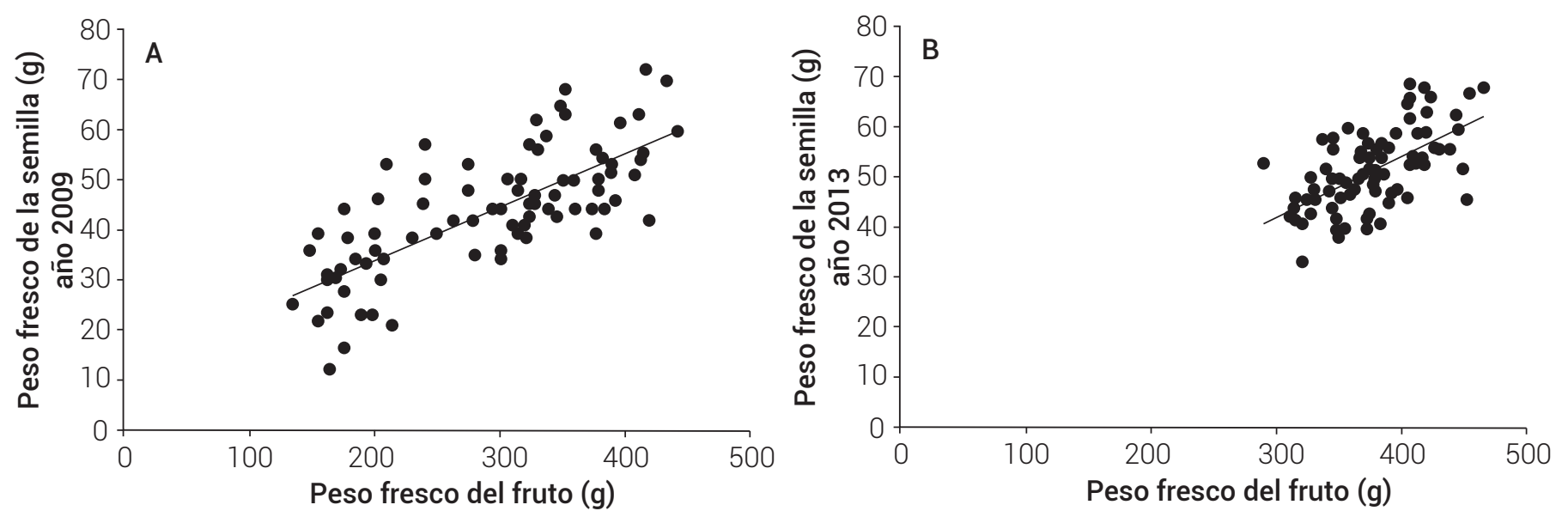

Figura 1. Relación entre el peso de la semilla y del fruto de aguacate Esther cosechados en las temporadas 2009 (A) con una $r=0.75$ y 2013 (B) con una $r=0.60$ en la localidad de Pedegua, Valparaíso, Chile.
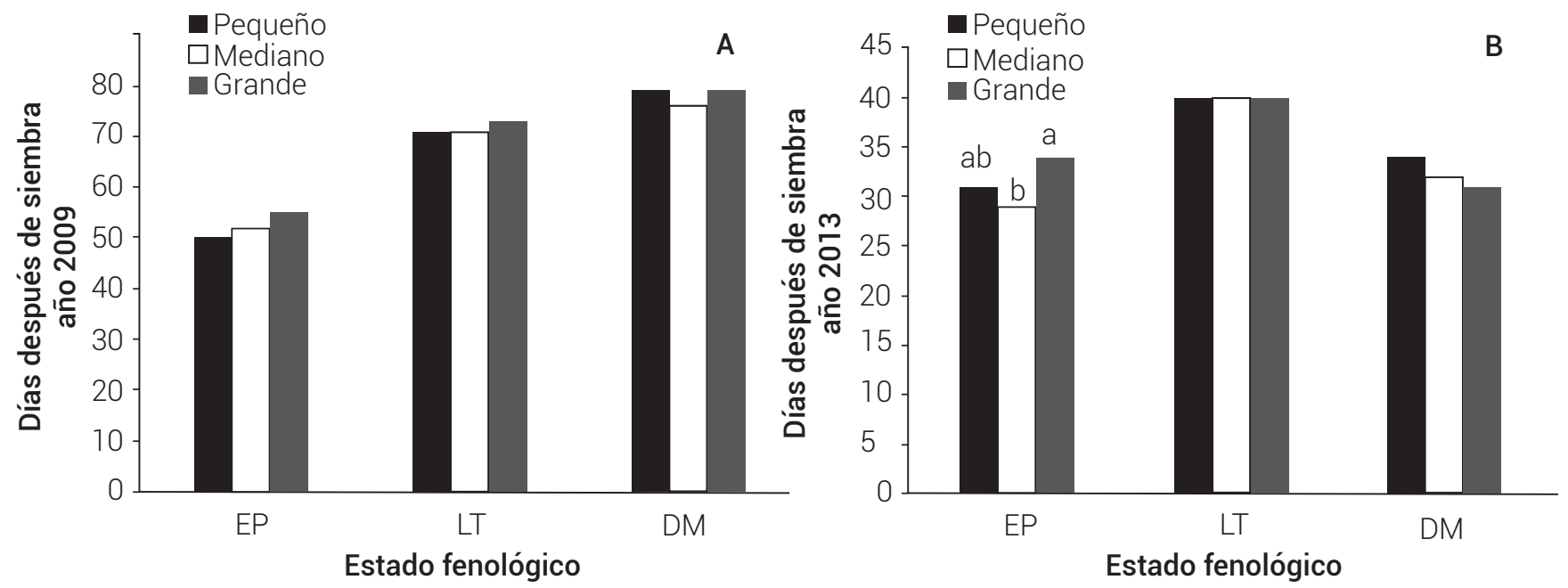

Figura 2. Tiempo promedio que tardaron en desarrollarse las plántulas de aguacate Esther en las temporadas 2009 (A) y 2013 (B). EP = emergencia de plúmula; $L T=$ tallo de $20 \mathrm{~cm}$ de longitud; $\mathrm{DM}=$ tallo de $4.5 \mathrm{~mm}$ de diámetro. Valparaíso, Chile.

relacionada con la fecha de recolección de las semillas, ya que en el 2009 se cosecharon en otoño frutos promedio de $291.6 \mathrm{~g}$ mientras que en el 2013 a fines de verano se cosecharon frutos de $375 \mathrm{~g}$. Según Cowan et al. (2001), las condiciones que controlan el proceso de división celular afectarían el desarrollo del fruto y su tamaño final, situación que puede explicar las diferencias aquí encontradas, aun cuando la causa misma requiera una revisión más detallada que no se encuentra en el ámbito de este estudio. Además, es importante considerar que el estado fisiológico del árbol es diferente en cada estación, lo que influye en la partición de fotoasimilados (Schaffer et al., 2013).

Barrientos-Priego et al. (1998) encontraron que el desarrollo de las plántulas de aguacate tiene una dependencia de los cotiledones durante su crecimiento inicial, sin con- siderar el tamaño de la semilla. Por su parte, Medina (2007; Com. pers. ${ }^{3}$ ) encontró escasa relación entre el tamaño de la semilla y el desarrollo inicial de las plántulas. En la presente investigación el tamaño de los cotiledones y del eje embrionario no afectó el desarrollo de las plántulas.

En Agave salmiana Vásquez et al. (2011) consideraron que la relación entre el tamaño de la semilla y el desarrollo de las plántulas existiría solo hasta el momento de la emergencia de las hojas cotiledonares, lo que en este caso con aguacate sucedió previo a las fechas de mediciones de las variables diámetro y longitud de tallo. En otras especies herbáceas se ha sugerido que el efecto del tamaño de las semillas sobre el desarrollo de la plántula estaría relacionado a la biomasa del embrión y de los cotiledones (Barrios et al., 2014; Peñaloza et al., 2005) y del contenido 

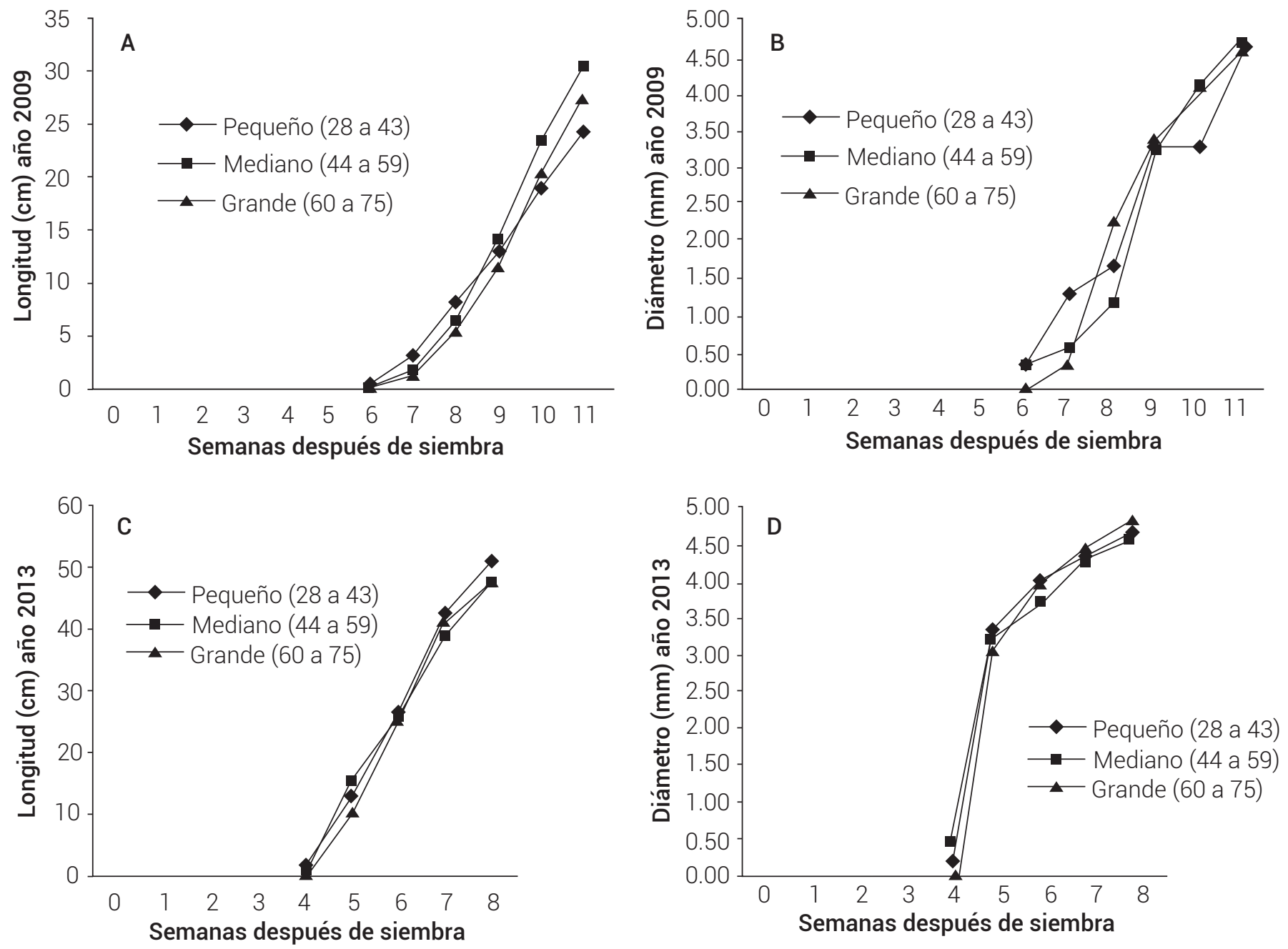

Figura 3. Crecimiento en longitud y diámetro de las plántulas de aguacate cv. Esther en las temporadas 2009 (A y B) y 2013 (C y, D). Valparaíso, Chile.

nutricional de las semillas (Sánchez et al., 2011).

\section{CONCLUSIONES}

Las semillas grandes (60 a $75 \mathrm{~g}$ ) de aguacate Esther se caracterizaron físicamente por tener mayor diámetro, perímetro de la semilla y peso fresco del eje embrionario, en comparación con las semillas pequeñas (28 a 43 g). El peso fresco del fruto se correlacionó positivamente con el peso fresco de la semilla. El peso fresco de la semilla utilizada en esta investigación demostró no tener efecto sobre el tiempo que tarda en desarrollarse plántulas al tamaño requerido para poder injertar.

\section{BIBLIOGRAFÍA}

AOSA, Association of Official Seed Analysts (1983) Seed Vigor Testing Handbook. AOSA, Springfield, IL., USA. 88 p.
Barrientos-Priego A. F., M. J. Morales-Nieto, G. Almaguer-Vargas, M. W. Borys, M. T. Martínez-Damián and F. Barrientos-Pérez (1998) Response of cotyledons detachment over the development of nursery seedlings of avocado (Persea americana Mill.). In: Proceedings of the World Avocado Congress III. Tel Aviv, Israel. 22-27 de Octubre. pp:211-216.

Barrios E., C. Lopez, J. Kohashi, J. Acosta, S. Miranda, N. Mayek y J. Canul (2014) Morfología del embrión en frijol y su comparación entre razas Durango y Jalisco. Revista Mexicana de Ciencias Agrícolas 5:965-978.

Bewley J., K. Bradford, H. Hilhorst and H. Nonogaki (2013) Seeds. Physiology of Development, Germination and Dormancy. 3rd. ed. Springer. New York, U.S.A. 399 p.

Blumenfeld A. and S. Gazit (1974) Development of seeded and seedless avocado fruits. Journal of American Society for Horticultural Science 99:442-448.

Calabrese F. (1992) El Aguacate. Ed. Mundi Prensa Libros S.A. Madrid, España. 246 p.

Castro M., C. Fassio, N. Darrouy y A. Ben Ya'acov (2003) Determinación de rangos de variabilidad en los niveles de producción del cv. Hass sobre portainjertos de semilla de raza mexicana en Chile. In: Proceedings V World Avocado Congress. Granada-Málaga, España. 19-24 de Octubre. pp:155-160.

Castro M. y C. Fassio (2013) Propagación Clonal de Paltos. Manual 
Técnico No. 1. Pontificia Universidad Católica de Valparaíso Facultad de Agronomía. Quillota, Chile. 23 p.

Ceballos A. y S. Montoya (2013) Evaluación química de la fibra en semilla, pulpa y cáscara de tres variedades de aguacate. Biotecnología en el Sector Agropecuario y Ambiental 11:103-112.

Cowan A., R. Cripps, E. Richings and N. Taylor (2001) Fruit size: Towards an understanding of the metabolic control of fruit growth using avocado as a model system. Physiologia Plantarum 111:127136.

Degani C., A. Goldring, I. Adato, R. El-Batsri and S. Gazit (1990) Pollen parent effect on outcrossing rate, yield, and fruit characteristics of 'Fuerte' avocado. HortScience 25:471-473.

Ernst A., A. Whiley and G. Bender (2013) Propagation. In: The Avocado. Botany, Production and Uses. B. Schaffer, N. Wolstenholme and A. Whiley (eds.). CABI. Oxfordshire, UK. pp:234-267.

Finazzo S., T. Davenport and B. Schaffer (1992) Partitioning of photoassimilates in avocado (Persea americana Mill.) during flowering and fruit set. Tree Physiology 14:153-164

Flores-Meza D., L. Vite-Cisneros and M. W. Borys (1990) Relations among the components of avocado seedlings (Persea americana Mill.). California Avocado Society Yearbook 74:207-214

García-Almañy M. y J. Lobo (2007) Influencia del tamaño de la semilla en la germinación y el crecimiento de las plántulas en tres variedades cubanas de tabaco negro. Cuba Tabaco 8:23-29.

García-Fajardo J. A., M. del R. Ramos-Godínez y J. Mora-Galindo (1999) Estructura de la semilla de aguacate y cuantificación de la grasa extraída por diferentes técnicas. Revista Chapingo Serie Horticultura 5:123-128

González V., R. Villar y R. Navarro (2008) Efecto del peso de la semilla y del progenitor en la biomasa y uso de las reservas de cuatro especies de Quercus. Cuadernos de la Sociedad Española de Ciencias Forestales 28:151-156.

ISTA, International Seed Testing Association (1995) Handbook of Vigour Test Methods. 3rd. ed. ISTA. Zurich, Switzerland. $119 \mathrm{p}$.

Lahav E. y U. Lavi (2015) Genética y mejoramiento. In: El Aguacate. Botánica, Producción y Usos. B Schaffer, N Wostenholme y A Whiley (eds.). CABI-Ediciones Universitarias de Valparaíso. Valparaíso, Chile. pp:73-112.

Laynez J.A., J. Méndez y J. Mayz (2007) Crecimiento de plántulas a partir de tres tamaños de semilla de dos cultivares de maíz (Zea mays L.), sembrados en arena y regados con tres soluciones osmóticas de sacarosa. IDESIA 25:21-36.

Olaeta J.A., M. Schwartz, P. Undurraga y S. Contreras (2007) Utilización de la semilla de palta (Persea americana Mill.) cv. Hass como producto agroindustrial. In: Proceedings VI World Avocado Congress (Actas VI Congreso Mundial del Aguacate) Viña Del Mar. 12-16 de Noviembre Viña del Mar, Chile. pp:1-9.
Ortega-David E., A. Rodriguez, A. David y A. Zamora-Burbano (2010) Caracterización de semillas de lupino (Lupinus mutabilis) sembrado en los Andes de Colombia. Acta Agronómica 57:111-118.

Peñaloza P., G. Ramírez-Rosales, M. Mcdonald and M. Bennett (2005) Lettuce (Lactuca sativa L.) seed quality evaluation using seed physical attributes, saturated salt accelerated aging and the seed vigour imaging system. Electronic Journal of Biotechnology 8:299-307.

Pérez C., A. Hernández, F. González, G. García, A. Carballo, T. Vásquez y M. Tovar (2006) Tamaño de semilla y relación con su calidad fisiológica en variedades de maíz para forraje. Agricultura Técnica 32:341-352.

Sánchez J., B. Muñoz, L. Montejo, M. Lescaille y R. Herrera (2011) Tamaño y nutrientes de semillas en 32 especies arbóreas de un bosque tropical siempreverde de Cuba y su relación con el establecimiento de las plántulas. Revista del Jardín Botánico Nacional 32-33:181-204.

Sánchez-Romero C., R. Perán-Quesada, A. Barceló-Muñoz, F. Pliego-Alfaro (2002) Variations in storage protein and carbohydrate levels during development of avocado zygotic embryos. Plant Physiology and Biochemistry 40:1043-1049.

Schaffer B., N. Wolstenholme and A. Whiley (eds.) (2013) The Avocado. Botany, Production and Uses. CABI, Oxfordshire, UK. 560 p.

Spann T.M. and L. Ferguson (2014) Commercial production of containergrown nursery trees: In: Citrus Production Manual. L. Ferguson and E. E. Grafton-Cardwell (eds.). University of California Agriculture and Natural Resources. Oakland, California. pp:107-116.

Sulewska H., K. Śmiatacz, G. Szymańska, K. Panasiewicz, H. Bandurska and R. Głowicka-Wołoszyn (2014) Seed size effect on yield quantity and quality of maize (Zea mays L.) cultivated in South East Baltic region. Zemdirbyste-Agriculture 101:35-40.

Tadeo-Robledo M., A. Espinosa-Calderón, R. Valdivia-Bernal, N. GómezMontiel, M. Sierra-Macías y B. Zamudio-González (2010) Vigor de las semillas y productividad de variedades de maíz. Agronomía Mesoamericana 21:31-38.

Vásquez E., J. García, C. Peña, H. Ramírez y V. Morales (2011) Tamaño de la semilla, emergencia y desarrollo de la plántula de maguey (Agave salmiana Otto ex Salm-Dyck). Revista Fitotecnia Mexicana 34:167-173.

Whiley A. and G. Anderson (2002) Study tour of South Africa, New Zealand, the UK and the USA (also incorporating Chile): milestone report for rootstock improvement for the avocado industry. Horticulture Australia. Sydney, Australia. 21 p.

Zhang C., K. Tanabe, H. Tani, H. Nacajima, M. Mori and E. Sakuno (2007) Biologically active gibberellins and abscisic acid in fruit of two late-maturing Japanese pear cultivars with contrasting fruit size. Journal of the American Society for Horticultural Science 132:452-458. 